\title{
Publisher's Note: Internal x-ray plateau in short GRBs: Signature of supramassive fast-rotating quark stars? [Phys. Rev. D 94, 083010 (2016)]
}

Ang Li®, Bing Zhang, Nai-Bo Zhang, He Gao, Bin Qi, and Tong Liu

(A) (Received 16 July 2020; published 29 July 2020)

DOI: 10.1103/PhysRevD.102.029902

This paper was published online on 28 October 2016 with the omission of a footnote label for the first author. Ang Li's footnote should read as "Corresponding author. liang@xmu.edu.cn". The paper has been corrected as of 21 July 2020. The paper is incorrect in the printed version of the journal. 